Egyptian

Orthodontic Journal

\title{
THE USE OF PEER ASSESSMENT RATING INDEX TO COMPARE TREATMENT OUTCOME IN EXTRACTION VERSUS NON-EXTRACTION TREATMENT
}

\author{
HANAN A. ISMAIL*
}

ABSTRACT

The PAR index have been developed to provide a single summary score for all the occlusal anomalies which may be found in a malocclusion. The difference in scores between the pre-and post-treatment cases reflects the degree of improvement and, therefore the success of treatment. The index was used in this study to assess treatment outcomes in 20 extraction and 20 non extraction class $I$ and II cases. Both groups showed good treatment outcomes however the percentage reduction in the PAR scores were higher in the non extraction group. The pretreatment PAR scores were significantly higher in the extraction group indicating more severe malocclusion at the start of treatment.

\section{INTRODUCTION}

The extraction of teeth for orthodontic purposes has always been a controversial subject in the orthodontic specialty. Early in the twentieth century Edward Angle believed that maintaining the whole set of the dentition in normal occlusion would allow normal masticatory function to cause jaw growth to accommodate the whole set of the dentition. This was argued by Calvin Case a few years later as he put the stability option in consideration. ${ }^{(1)}$ In the thirties many practitioners observed relapse with non-extraction treatments. Charles Tweed (1944) advocated four first premolar extraction to position the dentition upright on the supporting bone and initiated a favoring towards extraction therapy to provide stability on the long run for the treated cases. ${ }^{(2)}$

During the 1980s, the debate was raised again and favoring of non extraction returned until today. This is probably the result of many factors like early intervention and the use of functional appliance and the use of bonded brackets instead of fully banded appliances. Finally, a consumer driven market

\footnotetext{
* Assistant Prof. of Orthodontics, Faculty of Dentistry, Alexandria University.
} 
for treatment without extractions combined with the lack of experimental evidence to support either decision has ultimately kept the extraction and non extraction debate as a main orthodontic concern.

Paquette (1992) made a long term comparison between non extraction and premolar extraction in borderline class II cases. He found that although the two strategies produced significant long term difference in the convexity of the profile (non extraction group were two millimeters fuller), the two groups showed an essentially identical pattern of post treatment relapse settling that was related more to differential growth of the jaws than to the post treatment position and orientation of the denture. He concluded that his data provide little support for the concept of a single stable incisor angulation and to the troublesome claims that premolar extraction as opposed to expansion and bite jumping must of necessity produce distal mandibular displacement in the process of flattening the profile enough to ruin the face. ${ }^{(4)}$

As the extraction and non extraction debate continues, more objective information is needed to prevent the debate from hinging on the clinical experience of most orthodontists. In a specialty like ours the decision to extract or not should be based on scientific assessment of the treatment outcome.

The Peer Assessment Rating index (PAR) is used in grading orthodontic treatment results, which is self teaching and improves the quality of future treatment. The PAR index was developed to record malocclusion at any stage of treatment. The original PAR index ${ }^{(5)}$ consisted of 11 components of malocclusion which was reduced to 7 components after validation and weighting.

1- Upper anterior segment alignment: the recording zone is from the mesial anatomic contact point of the canine on one side to the mesial anatomic contact point of the canine on the other side.(displacement scores in table 1)

2- Lower anterior segment alignment.

3- Right buccal occlusion: the fit of the teeth is scored with respect to the three planes of space. The recording zone is from the canine to the last molar when the teeth are in occlusion. The antero-posterior, vertical and transverse irregularities are summed in table 2.

4- Over jet: positive over jet as well as teeth in cross bites are recorded (table 3).

5- Over bite: records vertical overlap and open bites of the anterior teeth. The recording zone includes the two lateral incisors. The tooth with the greatest overlap is recorded.(table 4).

6- Centre line: records centre line discrepancy in relation to the lower central incisors.(table 5). 
7- Left Buccal occlusion: the fit of the teeth is scored with respect to the three planes of space. The recording zone is from the canine to the last molar when the teeth are in occlusion. The antero-posterior, vertical and transverse irregularities are summed in table 2 .

A score of zero would indicate good alignment and higher scores (rarely beyond fifty) would indicate increased levels of irregularity. The overall score is recorded on the pre and post treatment dental casts and the difference between the scores represents the degree of improvement as a result of orthodontic intervention and active treatment. ${ }^{(5)}$

Table 1: Displacement scores

\begin{tabular}{|c|l|}
\hline Score & \multicolumn{1}{|c|}{ Discrepancy } \\
\hline 0 & $0 \mathrm{~mm}$ to $1 \mathrm{~mm}$ \\
1 & $1.1 \mathrm{~mm}$ to $2 \mathrm{~mm}$ \\
2 & $2.1 \mathrm{~mm}$ to $4 \mathrm{~mm}$ \\
3 & $4.1 \mathrm{~mm}$ to $8 \mathrm{~mm}$ \\
4 & Greater than $8 \mathrm{~mm}$ \\
5 & Impacted teeth \\
\hline
\end{tabular}

Table 2: Buccal occlusion assessments.

\begin{tabular}{|l|l|}
\hline \multicolumn{1}{|c|}{ Score } & \multicolumn{1}{c|}{ Discrepancy } \\
\hline Antero-posterior & \\
0 & Good interdigitation Class I,II and III \\
1 & Less than half unit discrepancy \\
2 & Half a unit discrepancy (cusp to cusp) \\
\hline Vertical & No discrepancy in intercuspation \\
0 & Lateral open bite on at least two teeth greater than 2 mm \\
1 & \\
\hline Transverse & No cross-bite \\
0 & Cross-bite tendency \\
1 & Single tooth in cross-bite \\
2 & More than one tooth in cross-bite \\
3 & More than one tooth in scissor bite \\
4 &
\end{tabular}


Table 3: Overjet measurements

\begin{tabular}{|l|l|}
\hline \multicolumn{1}{|c|}{ Score } & \multicolumn{1}{c|}{ Discrepancy } \\
\hline Overjet & $0-3 \mathrm{~mm}$ \\
0 & $3.1-5 \mathrm{~mm}$ \\
1 & $5.1-7 \mathrm{~mm}$ \\
2 & $7.1-9 \mathrm{~mm}$ \\
3 & Greater than $9 \mathrm{~mm}$ \\
4 & \\
\hline Anterior cross-bites & No discrepancy \\
0 & One or more teeth edge to edge \\
1 & One single tooth in cross-bite \\
2 & Two single tooth in cross-bite \\
3 & More than two teeth in cross-bite \\
4 &
\end{tabular}

Table 4: Overbite measurements Cross-bites including the canines are recorded in the anterior segment

\begin{tabular}{|c|c|}
\hline Score & Discrepancy \\
\hline \multicolumn{2}{|l|}{ Open bite } \\
\hline 0 & No open bite \\
\hline 1 & Open bite less than and equal to $1 \mathrm{~mm}$ \\
\hline 2 & Open bite $1.1-2 \mathrm{~mm}$ \\
\hline 3 & Open bite $2.1-3 \mathrm{~mm}$ \\
\hline 4 & Open bite greater than or equal to $4 \mathrm{~mm}$ \\
\hline \multicolumn{2}{|l|}{ Over bite } \\
\hline 0 & Less than or equal to one third coverage of the lower incisor \\
\hline 1 & Greater than one -third, but less than tow-thirds coverage of the \\
\hline 2 & lower incisor \\
\hline \multirow[t]{2}{*}{3} & Greater than two-thirds coverage of the lower incisor \\
\hline & Greater than or equal to full tooth coverage \\
\hline
\end{tabular}


Table 5: Centreline assessment

\begin{tabular}{|c|l|}
\hline Score & \multicolumn{1}{|c|}{ Discrepancy } \\
\hline 0 & Coincident and up to one-quarter lower incisor width \\
1 & One-quarter to one-half lower incisor width \\
2 & Greater than one-half lower incisor width \\
\hline
\end{tabular}

The PAR index have been used to evaluate treatment outcomes in various populations, ${ }^{(6-7)}$ to establish and maintain the highest standards of clinical excellence in orthodontics. The same index was also used to evaluate treatment protocols for class II malocclusion, that is the two phase approach versus the one phase plan. ${ }^{(8)}$

In this study the PAR index was used to assess treatment outcomes in non extraction and extraction protocols in an Egyptian population sample. The PAR index was shown in previous studies to be a valid and reliable method of assessing the outcome of orthodontic treatment by comparing the percentage PAR scores between both treatment strategies, this study can give a light to which is better finished. Is maximum intercuspation better after extraction or after non-extraction therapy? Are the teeth better aligned? Is the bite deep or not?

\section{Material and Methods}

The study sample consisted of 20 extraction (first premolars) and 20 non extraction cases collected from a single orthodontic clinic. Patients were between 13 and 18 years of age and had a class I or class II malocclusion at the beginning of treatment. Selected cases had full set of permanent dentition excluding the third permanent molar with no deciduous teeth left. Tooth agenesis or anomalies were absent.

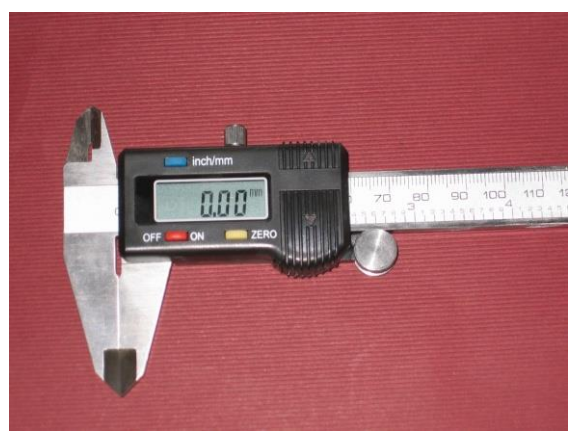

(Figure 1 ) Digital Caliper 
Treatment was completed using straight wire American Orthodontic brackets slot size 0.022 " 0.025 ". The immediate pretreatment and post treatment casts were collected and the PAR score was given to each cast using a digital caliper (figure1). Treatment time was 23 months $( \pm 6)$ for the non extraction treatment and 29 months $( \pm 4)$ for the extraction treatment.

The seven weighted components according to the British weightings are shown in the scoring record sheet which was used for every case (figure 2).

(Figure 2) PAR scoring sheet

Case number $(\quad)$

\section{PAR component}

Upper anterior segment Lower anterior segment

Right buccal occlusion
Antero-posterior ( ) Transverse posterior ( ) Vertical posterior ( )

Over jet (x6) ) cross bite (__

Overbite

Centre line

\section{Pre-treatment}

$(3-2)(2-1)(1-1)(1-2)(2-3)$

$(3-2)(2-1)(1-1)(1-2)(2-3)$

\section{Total}

Left buccal occlusion

Antero posterior ( ) Transverse posterior ( ) Vertical posterior ( )

Pre-treatment total

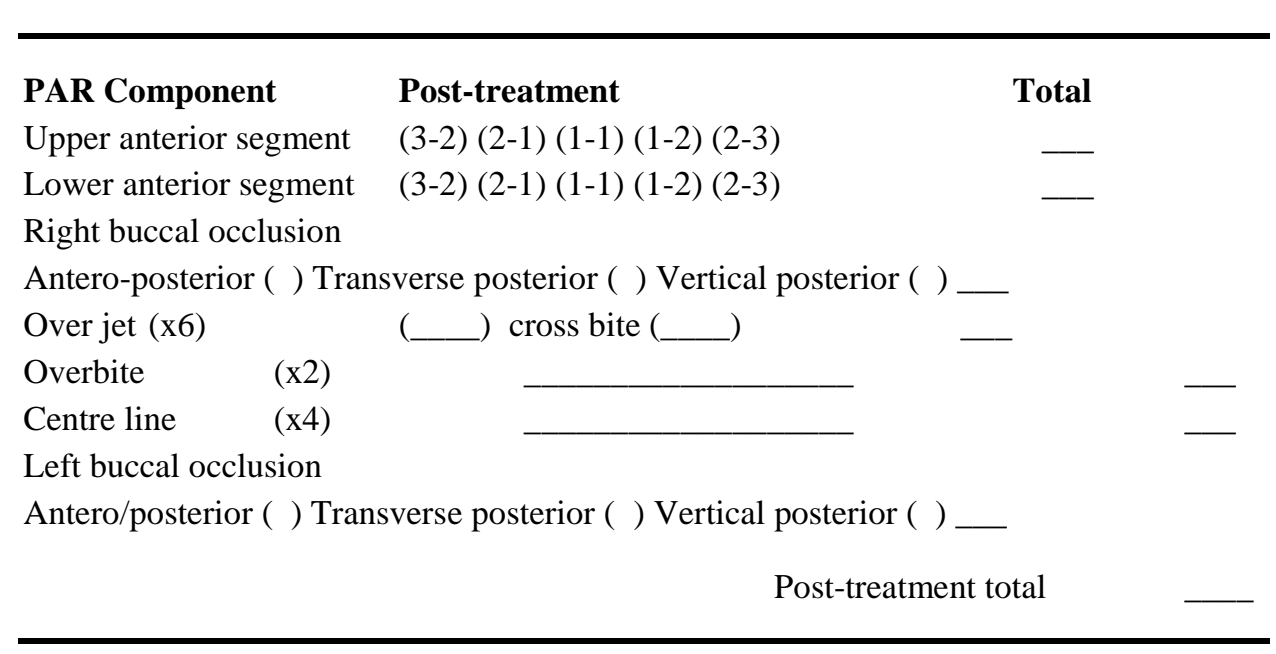

Change in PAR score ( ) \% change in PAR score ( ) 
A perfect occlusion would receive a score of zero, a score of one to nine indicates that good dental relationships are present and a score of forty indicates severe malocclusion. All study casts were scored by a single investigator using a digital caliper according to the weighted points described by Richmond to score each category of malocclusion.

The selected casts were scored according to the PAR criteria and weight for malocclusion severity and treatment difficulty using the weightings derived by the British validations. ${ }^{(5)}$

\section{Statistical analysis:}

Descriptive statistics were shown for different measurements as mean and standard deviation. Comparison of different measurements between before and after treatment was done using Wilcoxon signed ranks test. Percent change in values of measurements was computed as: [(pre treatment value- post treatment) / pre treatment] X 100. Contribution of each measurement in the total malocclusion score was calculated as: [score of measurement/ total malocclusion score] X 100 .

Comparison between extraction and non extraction cases as regards percent change in different measurements and in total score (pre and post treatment) was performed using t test or Mann Whitney $\mathrm{U}$ test for measurements that were not normally distributed.

Reliability was determined by rescoring the same casts after one month interval. Intraexaminer error was found negligible.

\section{RESULTS}

The mean and the standard deviation for each pretreatment component and posttreatment component that makes up the PAR score, the percentage reduction in the PAR score (\% RED) and the percentage contribution to the malocclusion for each component (\%MAL) are given in table 6 for the extraction group and in table 7 for the non extraction group. 
Egyptian

Orthodontic Journal

Table 6: Comparison between pre and post values in Extraction group:

\begin{tabular}{||l|c|c|c|c|c|c||}
\hline & $\begin{array}{c}\text { Pre } \\
\text { Treatment }\end{array}$ & $\begin{array}{c}\text { Post } \\
\text { Treatment }\end{array}$ & \% Reduction & \% Malocclusion & $\mathbf{Z}$ of Wsrt & P Value \\
\hline $\begin{array}{l}\text { Upper anterior segment } \\
\text { crowding }\end{array}$ & $4.80 \pm 3.00$ & $0 \pm 0$ & 100 & 15.99 & 3.74 & $<0.0001^{*}$ \\
\hline $\begin{array}{l}\text { Lower anterior segment } \\
\text { crowding }\end{array}$ & $4.05 \pm 2.46$ & $0.05 \pm 0.22$ & 95.00 & 13.89 & 3.84 & $<0.0001^{*}$ \\
\hline Right buccal occlusion & $1.75 \pm 1.65$ & $0.50 \pm 0.61$ & 83.11 & 5.56 & 3.30 & $0.001^{*}$ \\
\hline Over jet & $14.15 \pm 8.50$ & $1.50 \pm 2.67$ & 90.20 & 39.43 & 3.65 & $<0.0001^{*}$ \\
\hline Overbite & $2.50 \pm 1.93$ & $0.30 \pm 0.98$ & 90 & 7.20 & 3.38 & $0.001^{*}$ \\
\hline Centerline & $4.80 \pm 3.33$ & $0.55 \pm 1.28$ & 90 & 15.08 & 3.47 & $0.001^{*}$ \\
\hline Left buccal occlusion & $1.40 \pm 1.19$ & $0.55 \pm 0.51$ & 62.29 & 4.27 & 2.76 & $0.006^{*}$ \\
\hline
\end{tabular}

*: Statistically significant

The percentage reduction in malocclusion is statistically significant for all the components of the PAR index in extraction group.

Table 7: Comparison between pre and post values in NON EXTRACTION group:

\begin{tabular}{|l|c|c|c|c|c|c||}
\hline \hline & Pre treatment & Post treatment & $\%$ reduction & \% malocclusion & Z of Wsrt & P value \\
\hline $\begin{array}{l}\text { Upper anterior segment } \\
\text { crowding }\end{array}$ & $3.20 \pm 2.07$ & $0 \pm 0$ & 100 & 17.33 & 3.84 & $<0.0001^{*}$ \\
\hline $\begin{array}{l}\text { Lower anterior segment } \\
\text { crowding }\end{array}$ & $2.40 \pm 1.60$ & $0.10 \pm 0.31$ & 96.49 & 13.49 & 3.85 & $<0.0001^{*}$ \\
\hline Right buccal occlusion & $0.25 \pm 0.44$ & $0.05 \pm 0.22$ & 80 & 1.40 & 2.00 & $0.05^{*}$ \\
\hline Over jet & $8.70 \pm 6.30$ & $1.20 \pm 2.46$ & 87.50 & 38.94 & 3.49 & $<0.0001^{*}$ \\
\hline Overbite & $2.50 \pm 1.57$ & $0.10 \pm 0.45$ & 97.06 & 15.76 & 3.75 & $<0.0001^{*}$ \\
\hline Centerline & $2.00 \pm 2.05$ & $0 \pm 0$ & 100 & 9.63 & 3.16 & $0.002^{*}$ \\
\hline Left buccal occlusion & $0.70 \pm 0.92$ & $0 \pm 0$ & 100 & 3.44 & 2.72 & $0.006^{*}$ \\
\hline
\end{tabular}

*: Statistically significant

Volume 32 - December 2007 
The percentage reduction in malocclusion was statistically significant for all components of the PAR index in the nonextraction group.

Table 8: Comparison of difference between total pre and post PAR scores in extraction and non extraction groups:

\begin{tabular}{||l|c|c|c|c|c|c||}
\hline \multirow{2}{*}{} & \multicolumn{2}{|c|}{ Extraction } & \multicolumn{2}{c||}{ Non Extraction } & MWU & \multirow{2}{*}{ P Value } \\
\cline { 2 - 7 } & Mean \pm SD & Median & Mean \pm SD & Median & Test & \\
\hline Pre PAR score & $33.40 \pm 9.37$ & 36.00 & $19.75 \pm 7.93$ & 19.00 & 3.78 & $<0.0001^{*}$ \\
\hline Post PAR score & $3.45 \pm 3.09$ & 2.00 & $1.45 \pm 2.52$ & 0 & 3.07 & $0.002^{*}$ \\
\hline Difference & \multicolumn{2}{|c|}{$29.95 \pm 8.03$} & $18.30 \pm 6.65$ & & \\
\hline $\begin{array}{l}\text { Z of WSRT } \\
\text { P value }\end{array}$ & \multicolumn{2}{|c|}{3.92} & \multicolumn{2}{|c||}{3.92} & & \\
\hline
\end{tabular}

*: Statistically significant

Table 9: Comparison of percent change in PAR scores between extraction and non extraction groups:

\begin{tabular}{||l|c|c||}
\hline \multicolumn{1}{|c|}{ Percent Change } & Extraction & Non Extraction \\
\hline Mean \pm SD & $90.53 \pm 7.93$ & $94.14 \pm 9.61$ \\
\hline Median & 94.37 & 100 \\
\hline MWU test & & 2.39 \\
P value & $0.02 *$ \\
\hline
\end{tabular}

*: Statistically significant

Percentage reduction in PAR score was significantly higher for the non extraction group than for the extraction group. 
Table 10: Comparison of percent change in different dental parameters between extraction and non extraction cases:

\begin{tabular}{|c|c|c|c|c|}
\hline \multirow[t]{2}{*}{ Percent Change } & $\begin{array}{c}\text { Extraction } \\
\text { Percent Change }\end{array}$ & $\begin{array}{l}\text { Non Extraction } \\
\text { Percent Change }\end{array}$ & \multirow[t]{2}{*}{ T Test } & \multirow[t]{2}{*}{ P Value } \\
\hline & Mean \pm SD & Mean \pm SD & & \\
\hline Upper anterior segment crowding & $100 \pm 0$ & $100 \pm 0$ & - & - \\
\hline Lower anterior segment crowding $\mathbb{I}$ & $95.00 \pm 22.36$ & $96.49 \pm 11.89$ & 0.60 & $0.55 \mathrm{NS}$ \\
\hline Right buccal occlusion & $83.11 \pm 20.98$ & $80.00 \pm 44.72$ & 0.22 & $0.83 \mathrm{NS}$ \\
\hline Over jet & $90.20 \pm 16.47$ & $87.50 \pm 26.87$ & 0.35 & $0.73 \mathrm{NS}$ \\
\hline Overbite II & $90.00 \pm 28.03$ & $97.06 \pm 12.13$ & 0.75 & $0.46 \mathrm{NS}$ \\
\hline Centerline II & $90.00 \pm 18.42$ & $100.00 \pm 0$ & 1.74 & $0.08 \mathrm{NS}$ \\
\hline Left buccal occlusion $\mathbb{I}$ & $62.29 \pm 41.84$ & $100.00 \pm 0$ & 2.68 & $0.007 *$ \\
\hline
\end{tabular}

II: Mann Whitney test used

Percentage reduction in the PAR score was only significant in the left buccal occlusion when comparing extraction and non extraction cases.

Table 11: Comparison between extraction and non extraction group as regards pre treatment measurements:

\begin{tabular}{||l|c|c|c|c||}
\hline \hline & Extraction & Non Extraction & \multirow{2}{*}{ Z of MWUI } & \multirow{2}{*}{ P Value } \\
\cline { 2 - 5 } & Mean \pm SD & Mean \pm SD & & \\
\hline Upper anterior segment crowding & $4.80 \pm 3.00$ & $3.20 \pm 2.07$ & 1.79 & $0.07 \mathrm{NS}$ \\
\hline Lower anterior segment crowding & $4.05 \pm 2.46$ & $2.40 \pm 1.60$ & 2.33 & $0.02^{*}$ \\
\hline Right buccal occlusion & $1.75 \pm 1.65$ & $0.25 \pm 0.44$ & 3.92 & $<0.0001^{*}$ \\
\hline Over jet & $14.15 \pm 8.50$ & $8.70 \pm 6.30$ & 2.22 & $0.03^{*}$ \\
\hline Overbite & $2.50 \pm 1.93$ & $2.50 \pm 1.57$ & 0.04 & $0.97 \mathrm{NS}$ \\
\hline Centerline & $4.80 \pm 3.33$ & $2.00 \pm 2.05$ & 2.75 & $0.006^{*}$ \\
\hline Left buccal occlusion & $1.40 \pm 1.19$ & $0.70 \pm 0.92$ & 2.12 & $0.03^{*}$ \\
\hline
\end{tabular}

II: Mann Whitney test

NS: Not statistically significant

*: Statistically significant 
The PAR scores were significantly higher in the extraction group except in upper anterior segment crowding and overbite.

Table 12: Comparison between extraction and non extraction group as regards post treatment measurements:

\begin{tabular}{||l|c|c|c|c||}
\hline & Extraction & Non Extraction & $\begin{array}{c}\text { Z of } \\
\text { MWUII }\end{array}$ & \multirow{2}{*}{ P Value } \\
\cline { 2 - 3 } & Mean \pm SD & Mean \pm SD & 0 & $1.00 \mathrm{NS}$ \\
\hline Upper anterior segment crowding & $0 \pm 0$ & $0 \pm 0$ & 0.59 & $0.55 \mathrm{NS}$ \\
\hline Lower anterior segment crowding & $0.05 \pm 0.22$ & $0.10 \pm 0.31$ & 2.89 & $0.004^{*}$ \\
\hline Right buccal occlusion & $0.50 \pm 0.61$ & $0.05 \pm 0.22$ & 0.37 & $0.71 \mathrm{NS}$ \\
\hline Overjet & $1.50 \pm 2.67$ & $1.20 \pm 2.46$ & 0.62 & $0.53 \mathrm{NS}$ \\
\hline Overbite & $0.30 \pm 0.98$ & $0.10 \pm 0.45$ & 2.08 & $0.04 *$ \\
\hline Centerline & $0.55 \pm 1.28$ & $0 \pm 0$ & 3.85 & $<0.0001^{*}$ \\
\hline Left buccal occlusion & $0.55 \pm 0.51$ & $0 \pm 0$ & & \\
\hline
\end{tabular}

II: Mann Whitney test

NS: Not statistically significant

*: Statistically significant

The individual improvement in the PAR scores were statistically higher in the non-extraction group for the buccal occlusion and center line.

\section{DISCUSSION}

This study was designed to assess difference in dental parameters between patients treated with first premolar extractions and patients treated without extractions. Changes in the dental parameters were measured using the percentage reduction in the weighted PAR scores.

The British weighting system ${ }^{(5)}$ was used in this study.The peer assessment rating (PAR) index was specifically designed to provide a more objective assessment of treatment success. The PAR index has been shown to have good intra- and interexaminer reliability, and it has been used in several studies. ${ }^{(5,9,10)}$

Previous studies ${ }^{(5,9)}$ have shown that $70 \%$ reduction in PAR scores can be considered a great improvement in occlusal factors. In this study the extraction group showed $90.53 \%$ mean percentage reduction in the PAR score and the nonextraction group showed $94.14 \%$ mean reduction. Non of the patients treated in either groups had a percentage reduction in the PAR score less than $70 \%$ indicating that both groups had good to excellent treatment outcomes. The 
percentage reduction in the PAR was significantly higher in the nonextraction sample indicating better treatment outcome in the nonextraction sample. Comparing these results to a similar study done by Holmanj.k.et $\mathrm{al}^{(3)}$ the percentage reduction in the PAR scores were comparable in both extraction and non extraction samples in their study and the percentage reduction in PAR scores was lower than this study with a mean reduction of $78.59 \%$ reduction. The difference in the results is probably due to the larger sample selected (100 extraction and 100 non extraction cases) over a long period of time (1981to 1995), there is a common belief that operators improve with time. Also the sample contained all classes of malocclusion and treatment protocols varied. However treatment was done by one specialist as in this study.

A recent study done to evaluate treatment changes and quality of finishing in 94 class 1 patients ${ }^{(10)}$ treated with four premolars extraction using the PAR index showed a 78.54\% mean PAR reduction and treatment showed a good standard of finishing. The PAR reduction seems to be much lower than in our study probably because their sample was treated by several orthodontic graduate students.

Woods et $\mathrm{al}^{(11)}$ evaluated 65 patients representing several types of malocclusion treated various treatment protocols and found $85.6 \%$ mean PAR reduction with treatment. Their percentage reduction is comparable to ours, although slightly less could be due to the larger sample and different types of malocclusion. The treatment was done by a single specialist like in this study.

Comparing the mean percentage reduction for each dental parameter, the percentage reduction was similar in both extraction and non extraction groups and the PAR reduction was insignificant except for the left buccal occlusion which was $62 \%$ average reduction in the extraction group and $100 \%$ reduction in the non extraction group. The post treatment PAR scores were significantly lower in the non extraction group.

However when comparing the pre treatment PAR scores in both groups, the pre PAR scores were significantly higher (33.4) in the extraction group indicating more severe malocclusion compared to the non extraction group (19.75).

The Mann Whitney test showed that five out of the seven components of the PAR index (LAS, RBO,OJ, CL, LBO) had mean pretreatment PAR sores significantly greater in the extraction sample indicating more severe dental malocclusions concerning those parameters. That is to say premolar extraction was the treatment of choice in cases with lower incisor crowding, more severe 
buccal segment malocclusion, greater over jet and large midline deviations. Both upper anterior segment crowding and overbite did not differ significantly between both groups in fact the overbite scores were almost similar in both groups. The results of this study shows similarity to the results of Holman et al (3) who found that the pretreatment scores for the extraction cases were higher concerning the LAS, RBO, OJ,CL, LBO. Interestingly he found the overbite not only insignificantly different between both groups but even slightly higher in the non extraction group. He concluded that increased over bite component is a measure for decreased lower facial height and low angle indicating that the high angle cases were in the extraction sample. In some cases extraction is necessary to close open bites whereas many deep bites cases are completed without extracting teeth. Therefore the scores of the different components of the PAR index confirm statistically to what is felt clinically.

In this study the overbite pretreatment scores were similar in both groups could be attributed to the size and quality of the sample.

The significant increase in the pretreatment scores concerning the over jet and lower dental crowding was consistent with Paquette et $\mathrm{al}^{(4)}$ who reported that the discriminant function used to identify their group of border line extraction cases included measures of dental crowding and over jet.

It is clear that there is an interaction between the initial severity of malocclusion and the extraction decision. Of course extraction decision should be based on factors other than the dental relation as the profile of the patient and the skeletal configuration of the case; however in borderline cases where the decision to extract or not opens the endless debate again the previous findings can be put in consideration. The choice of non extraction treatment for border line cases reduces treatment time and gives better treatment outcomes as concluded from the results of this study, as treatment mechanics include leveling and alignment and finishing stages. There is no retraction and space closure plus more complicated finishing procedures to adjust the contact between the second premolars and the canines which is never as perfect as the occlusion of the whole set of the dentition.

When comparing the percentage reduction in PAR scores between both groups concerning individual dental parameters it was found that the only significant PAR reduction was in the left buccal occlusion which was significantly improved in the non extraction group than the extraction group.

While there are many benefits in the use of the PAR index, numerous limitations must be remembered. The index measures only dento-occlusal 
changes and does not take into account changes in the soft tissues that result from orthodontic treatment.

The index measures only study models and does not account for decalcification, gingival recession, root resorption, or TMJ considerations that may result from orthodontic treatment. The study used pre and post treatment casts, so long term stability wasn't measured. Also other diagnostic aids as cephalograms and photographs were not used.

\section{CONCLUSION}

1- Percentage reduction in PAR scores were higher in non extraction cases.

2- High pretreatment PAR scores were present in the extraction cases indicating more severe malocclusions.

3- Extraction cases had higher PAR scores for the over jet, lower anterior crowding, buccal occlusion and mid line discrepancy.

\section{REFERENCES}

1. Case CS. The question of extraction in orthodontia. Am J Orthod 1964; 50:660-691.

2. Tweed $\mathrm{CH}$. Indications for extraction of teeth in orthodontic procedure. Am J Orthod 1944;20:405-425.

3. Holman JK, Hans MG, Nelson S, Powers MP. An assessment of extraction versus non extraction treatment using the peer assessment rating (PAR) index. The Angle Orthod 1998;68:527-534.

4. Paquette DE, Beattie JR, Johnston LE Jr. A long term comparison of non extraction and premolar extraction edgewise therapy in borderline Class II patients. Am J Orthod Dentofacial Orthop 1992;102:1-14.

5. Richmond S, Shaw WC, O'Brien KD, Buchanan IB, Jones R, Stephens CD, Roberts CT, Andrews M. The development of the PAR index (Peer Assessment Rating): reliability and validity.Eur J Orthod 1992;14:125-39.

6. Cadman KS, Clover KE, Heo G, Warren S and Major PW. Orthodontic treatment outcome in a first nations population in Alberta Canada: A comparative study. Am J Orthod Dentofacial Orthop 2002;121:396-402. 
7. Dyken RA, Sadowsky PL, Hurst D. Orthodontic outcomes assessment using the Peer Assessment Rating index. The Angle Orthod. 2001;71:164-169.

8. King GJ, McGorray SP, Wheeler TT, Dolu C, Taylor M. Comparison of Peer Assessment Ratings (PAR) from one phase and two phase treatment protocols for class II malocclusion. Am J Orthod and Dentofacial Orthop 2003; 123:489-496.

9. Deguzman L, Bahiraei D, Vig KWL, Vig PS, Weyant RJ and O'BrienKO. The validation of the Peer Assessment Rating index for malocclusion severity and treatment difficulty. Am J Orthod and Dentofacial Orthop 1995; 107:172-176.

10. Freitas KMS, Freitas DS, Valarelli FP, Freitas MR, Janson G. PAR evaluation of treated class I extraction patients. The Angle Orthod 2007;78:270-274.

11. Woods M, Lee D, Crawford E. Finishing occlusion, degree of stability and PAR index. Aust Orthod J 2000;16:9-15. (quoted from 10) 